\title{
NMR-Based Encoding and Processing of Alphanumeric Information (POSTER)
}

\author{
Tamar Ratner ${ }^{1}$, Ofer Reany ${ }^{1}$, and Ehud Keinan ${ }^{1,2}$ \\ ${ }^{1}$ The Schulich Faculty of Chemistry and Institute of Catalysis Science and Technology, \\ Technion - Israel Institute of Technology Technion City, Haifa 32000, Israel \\ ${ }^{2}$ Departments of Molecular Biology and Cell Biology, and the Skaggs Inst. of \\ Chemical Biology, 10550 North Torrey Pines Road, La Jolla, CA 92037, USA \\ keinan@techunix.technion.ac.il
}

\begin{abstract}
We present here a novel experimental computing device that is based on ${ }^{1} \mathrm{H}$ Nuclear Magnetic Resonance (NMR) readout of chemical information. This chemical encoding system utilizes two measurable parameters of homogeneous mixtures, chemical shift and peak integration, for different applications: 1) text encoding device that is based on spectral representation of a sequence of symbols, 2) encoding of 21-digit binary numbers, each represented by an NMR spectrum, and their algebraic manipulations, such as addition and subtraction, and 3) encoding of 21-digit decimal numbers.
\end{abstract}

We have selected 22 compounds, each characterized by a simple ${ }^{1} \mathrm{H}$ NMR spectrum with a single absorption within the chemical shift range of $\delta 2-4 \mathrm{ppm}$. The use of NMR allows us to detect the presence / absence of each substances, as well as its relative concentration, thus creating a two dimensional matrix of two parameters.

The first application enables molecular information storage and encryption. The relative concentration of each component, as measured by the relevant peak integration, can represent a symbol. Integration of any of the 22 compounds in our library can be accurately measured for at least 10 different levels of concentrations. In order to represent 26 letters with high precision and also increase the encryption complexity we assigned each symbol to a pair of consecutive peaks in the spectrum. Based on 10 concentration levels of each component, a pair of peaks can produce $10^{2}=100$ combinations, each representing a different symbol.

A second application of this system, in addition to its obvious memory abilities, enables mathematical operations. The NMR spectrum of a 22component mixture represents a 21-digit binary number (on of the peaks served as an integration reference) where each of the peaks encodes for a specific digit. In any of the input mixtures (numbers) each compound was either present or absent, representing either 1 or 0 , respectively. This encoding can produce as many as $2^{21}=2,097,152$ different numbers. We used the various binary numbers to carry out addition operations by combining two or more solutions (numbers). Electronically, subtraction operations were also preformed.

A third application is the representation of decimal numbers. As before, each of the peaks encodes for a specific digit. In any of the input mixtures each compound was present in one of 10 different relative concentrations, representing the 10 digits of the decimal number. 